\title{
Avaliação da capacidade de adsorção do corante azul de metileno em soluçãoes aquosas em caulinita natural e intercalada com acetato de potássio
}

\section{(Evaluation of the capacity of adsorption of methylene blue die in aqueous solutions in natural kaolinite and intercalated with potassium acetate)}

\author{
S.P. Oliveira ${ }^{I}$, W. L. L.Silva ${ }^{I}, R . R$. Viana $^{I}$ \\ ${ }^{\text {I} P r o g r a m a ~ d e ~ P o ́ s-G r a d u a c ̧ a ̃ o ~ e m ~ G e o c i e ̂ n c i a s, ~ U n i v e r s i d a d e ~ F e d e r a l ~ d e ~ M a t o ~ G r o s s o, ~ F e r n a n d o ~ C o r r e ̂ a, ~ s / n, ~}$ \\ 78060 900, Cuiabá, MT, Brasil
}

\begin{abstract}
Resumo
O presente estudo teve como objetivo avaliar a capacidade de adsorção do corante azul de metileno em soluções aquosas em caulinita natural proveniente da região de Bom Jardim de Goiás, GO e na sua forma intercalada com o composto orgânico acetato de potássio. As amostras natural e intercalada foram caracterizadas por difração e fluorescência de raios X, microscopia eletrônica de varredura e infravermelho. O processo de intercalação resultou em um aumento do espaçamento basal $\left(\mathrm{d}_{001}\right)$ na estrutura do argilomineral, que pode ser verificado pela difração de raios X. A habilidade das amostras para remover o corante azul de metileno de soluções aquosas foi investigada através do método de batelada, cujos dados foram calculados pelo método não-linear através dos modelos de Langmuir, Freundlich e Sips. O modelo que melhor se ajustou aos dados experimentais foi o de Sips, para ambas as amostras. Para o estudo cinético três modelos foram testados, pseudo-primeira ordem, pseudo-segunda ordem e Avrami. Para ambas as amostras o modelo de Avrami foi o que melhor se ajustou aos dados experimentais. A capacidade máxima de adsorção atingida pela amostra intercalada foi de 79,34 mg.g $\mathrm{g}^{-1} \mathrm{e}$ para natural foi de 72,14 mg. $\mathrm{g}^{-1}$. Estes valores mostram que as amostras de caulinita natural e intercalada podem ser consideradas como bons adsorventes, na remoção do corante azul de metileno em meios aquosos. Palavras-chave: caulinita, intercalação, acetato de potássio, adsorção, azul de metileno.
\end{abstract}

\begin{abstract}
The present study aimed to evaluate the adsorption capacity of methylene blue dye in aqueous solutions by natural kaolinite from the region of Bom Jardim de Goiás, GO, Brazil, and its form intercalated with the organic compound potassium acetate. The natural sample and intercalated were characterized by diffraction and X-ray fluorescence, scanning electron microscopy and infrared. The intercalation process resulted in an increase in the basal spacing $\left(d_{001}\right)$ in the clay mineral structure, which may be verified by $X$-ray diffraction. The capacity of samples to remove methylene blue from aqueous solutions was investigated by the batch method and the data were calculated by the non-linear method using model the Langmuir, Freundlich and Sips. The model that best fitted the experimental data was the Sips for both samples. For kinetic studies have been tested three models, pseudo-first order, pseudosecond order, and Avrami. For both samples the Avrami model was the best fit to the experimental data. The maximum adsorption capacity reached by the intercalated sample was $79.34 \mathrm{mg} \cdot \mathrm{g}^{-1}$ and natural was of $72.14 \mathrm{mg} \cdot \mathrm{g}^{-1}$. These values show that natural and intercalated kaolinite may be considered good adsorbents to the removal of methylene blue dye in aqueous medium.
\end{abstract}

Keywords: kaolinite, intercalation, potassium acetate, adsorption, methylene blue.

\section{INTRODUÇÃO}

Corantes provenientes de processos industriais, como fabricação de papel, indústria têxtil, galvanoplastia, fábrica de celulose, produção de alimentos e cosméticos são as principais fontes de poluição da água. As complexas estruturas aromáticas e as propriedades xenobióticas dos corantes os tornam difíceis de serem degradados [1]. O azul de metileno (MB) é um corante catiônico, e possui uma variedade de aplicações, sendo utilizado no tingimento de algodão, lãs e papel, tinturas temporárias para cabelos, etc. Devido à sua forte adsorção em suportes sólidos, o azul de metileno, muitas vezes, serve como um composto modelo para a remoção de corantes e de contaminantes orgânicos a partir de soluções aquosas [2]. Ainda que o azul de metileno não seja tóxico quanto aos metais pesados, a exposição aguda pode causar efeitos prejudiciais à saúde como aumento do batimento cardíaco, dor de cabeça intensa, náuseas, vômitos, diarreia e necrose do tecido humano. Muitos métodos físicos e químicos são empregados para o tratamento de efluentes contendo corantes, tais como, adsorção, eletroquímica, precipitação, filtração, ozonização entre outros, sendo, no entanto, a adsorção o processo de tratamento mais utilizado [3]. O carvão ativado é o adsorvente mais utilizado comercialmente neste método, porém seu uso é limitado devido ao seu alto custo [4]. A constante busca por novos 
materiais de baixo custo em substituição ao carvão ativado é crescente e justificável. Avanços na preparação de materiais híbridos orgânico-inorgânicos por intercalação de moléculas orgânicas em caulinita representam a possibilidade de desenvolvimento de novos e interessantes materiais [5]. A caulinita é um argilomineral amplamente estudado e conhecido por suas características físico-químicas e versáteis aplicações industriais. Dependendo da aplicação, muitas vezes, a caulinita é modificada a partir do seu estado natural, por tratamentos físicos ou químicos com o intuito de melhorar as propriedades do material [6].

O objetivo do presente estudo foi avaliar o desempenho da caulinita natural e de sua forma intercalada no processo de adsorção do corante azul de metileno em soluções aquosas. A matriz modificada foi obtida através da intercalação da caulinita natural com acetato de potássio. O desenvolvimento do estudo do equilíbrio de adsorção foi realizado através dos modelos de Langmuir, Freundlich e Sips, e o estudo cinético foi obtido pelos modelos de pseudo-primeira ordem, pseudo-segunda ordem e Avrami. Ambos os estudos foram realizados pelo método não-linear.

\section{MATERIAIS E MÉTODOS}

\section{Adsorbato}

A solução estoque do corante azul de metileno (Synth Produtos Químicos, 95\%) foi preparada pela dissolução do corante em água deionizada na concentração de 5020 mg. $\mathrm{L}^{-1}$. As soluções de trabalho foram obtidas por diluição da solução estoque. Todas as soluções foram preparada com água deionizada (18 M $\Omega . c m)$, obtido a partir de uma purificação do sistema de água MilliQ.

\section{Material original}

A amostra de argila utilizada neste trabalho foi coletada em uma antiga pedreira denominada Granito Serra Verde na região de Bom Jardim de Goiás, GO. Inicialmente a amostra argilosa "in natura" foi beneficiada em um jogo de peneiras ABNT, cujo material utilizado para os ensaios foi o passante da peneira de 170 mesh (granulometria inferior a $0,088 \mathrm{~mm}$ ). Este material foi caracterizado e posteriormente intercalado com acetato de potássio P.A (Vetec Química Fina Ltda.).

\section{Métodos de caracterização}

Para a obtenção de difratogramas de raios X (DRX) foi utilizado o método do pó em um difratômetro Shimadzu XRD-6000 com radiação Cu-k $\alpha(\lambda=1,54 \AA$ ) , monocromador, $40 \mathrm{kV}, 30 \mathrm{~mA}$. A varredura de $2 \theta$ foi feita na faixa de 2 a $30^{\circ}$.

As análises químicas foram pela técnica de fluorescência de raios $\mathrm{X}$ com detector de energia dispersiva (EDS). O equipamento utilizado foi Shimadzu EDX-700HS, com um tempo de aquisição $200 \mathrm{~s}$ por canal analítico.

As micrografias foram obtidas por microscopia eletrônica de varredura (MEV)em um microscópio Shimadzu Superscan
Scanning SSX-550. As amostras foram depositadas em um porta-amostra com fita adesiva de carbono coberta com uma fina camada de ouro. As condições de análise foram feitas com tensão de $15 \mathrm{kV}$ com ampliações de 700 e 1000 vezes.

Os espectros de absorção na região do infravermelho foram obtidos em um espectrômetro Varian 660-IR Series, com transformada de Fourier (FTIR). As amostras foram embutidas em pastilhas de $\mathrm{KBr}$ com $1 \%$ de amostra e a leitura foi na região de $4000 \mathrm{a} 400 \mathrm{~cm}^{-1}$.

\section{Intercalação da amostra}

Uma mistura de $8,0 \mathrm{~g}$ da amostra natural e $3,4 \mathrm{~g}$ de acetato de potássio foi moída/misturada manualmente por $15 \mathrm{~min}$ em gral de ágata. Posteriormente essa mistura foi moída/misturada por $2 \mathrm{~h}$ em moinho automático de cuba e bolas de ágata (Fritsch Pulverisette 6). A mistura que se encontrava parcialmente úmida, devido à higroscopicidade do acetato, foi deixada em estufa por $1 \mathrm{~h}$ a $100{ }^{\circ} \mathrm{C}$ e então foi adicionada $1,10 \mathrm{~mL}$ de água destilada, e novamente moída/ misturada manualmente por mais $15 \mathrm{~m}$. Ao final a mistura foi deixada em estufa a $40{ }^{\circ} \mathrm{C}$ por $24 \mathrm{~h}$. [7]

\section{Determinação da adsorção do corante azul de metileno}

Inicialmente foi feita uma investigação prévia para verificar qual o $\mathrm{pH}$ que melhor adsorveria o azul de metileno; a adsorção foi verificada na faixa de $\mathrm{pH} 2,0$ a 10 . O ajuste do $\mathrm{pH}$ foi feito pela adição de soluções de $\mathrm{NaOH}$ e $\mathrm{HCl}$ (SigmaAldrich) a 0,10 mol. $\mathrm{L}^{-1}$, em um pHmetro Hanna 21 equipado com eletrodo de vidro Hanna (HI 1110B).

A determinação da adsorção do corante em solução foi feita pelo método de batelada, em que massa de $0,2 \mathrm{~g}$ das amostras natural e intercalada foi dissolvida em $20,0 \mathrm{~mL}$ de água deionizada em frascos de polietileno; tal sistema recebeu alíquotas do corante que variaram de 0,25 a 7,0 mL. Posteriormente, o volume de cada frasco foi ajustado para 40,0 mL obtendo-se concentrações do corante na faixa de 31,37 a $878,50 \mathrm{mg} \cdot \mathrm{L}^{-1}$. Em seguida os frascos foram agitados por $35 \mathrm{a} 400 \mathrm{~min}$ em temperatura de $25 \pm 1^{\circ} \mathrm{C}$. Os adsorventes foram separados das soluções aquosas por centrifugação a $2400 \mathrm{rpm}$ por $10 \mathrm{~min}$. As concentrações finais do corante azul de metileno remanescentes nas soluções foram determinadas por espectrofotometria na faixa do visível em um espectrofotômetro BEL Photonics SP-2000 UV. Para a leitura, os sobrenadantes foram diluídos para adequar sua concentração à janela de leitura do equipamento.

As medidas de absorbância foram feitas no comprimento de onda máximo do corante MB (660 nm). A quantidade do corante MB adsorvida pelos adsorventes foi calculada pela aplicação da equação A [8].

$$
\mathrm{q}_{\mathrm{e}}=\frac{\left(\mathrm{C}_{0}-\mathrm{C}_{\mathrm{f}}\right) \cdot \mathrm{V}}{1}
$$

na qual $\mathrm{q}_{\mathrm{e}}$ é a quantidade adsorvida do corante pelo adsorvente $\left(\mathrm{mg} \cdot \mathrm{g}^{-1}\right), \mathrm{C}_{0}$ e $\mathrm{C}_{\mathrm{f}}$ são correspondentes as concentrações inicial e final do corante, respectivamente (mg. $\left.\mathrm{L}^{-1}\right)$, $\mathrm{m}$ é a massa do 
adsorvente (g) e V é o volume da solução do corante em contato com o adsorvente (L).

\section{Modelos de equilíbrio e cinéticos de adsorção}

O equilíbrio de adsorção foi estudado através dos modelos de Langmuir [9], Freundlich [10] e Sips [11]. A cinética de adsorção do sistema adsortivo foi estudada através dos modelos de pseudo-primeira ordem [12], pseudo-segunda ordem [13] e Avrami [14].

\section{RESULTADOS E DISCUSSÃO}

\section{Caracterização das amostras}

Os resultados da difração de raios $\mathrm{X}$ das amostras natural e intercalada estão apresentados nas Figs. 1a e 1b, respectivamente. Estes resultados mostraram que a amostra natural (Fig. 1a) é um argilomineral impuro, com a presença de caulinita caracterizada pelas distâncias interplanares 7,16 Å e 3,56 ̊. Existe ainda em teores mais baixos a presença de ilita, caracterizada pelas distâncias interplanares 10,16 , $4,99 \AA ̊$ e 3,36 A e quartzo, caracterizado pelas distâncias 4,26 e 3,36 ̊. Valores similares a estas distâncias interplanares podem ser encontrados [15].

Após a intercalação, observou-se uma mudança nos padrões de difração. O pico em $12,33^{\circ}(2 \theta)$ da caulinita,

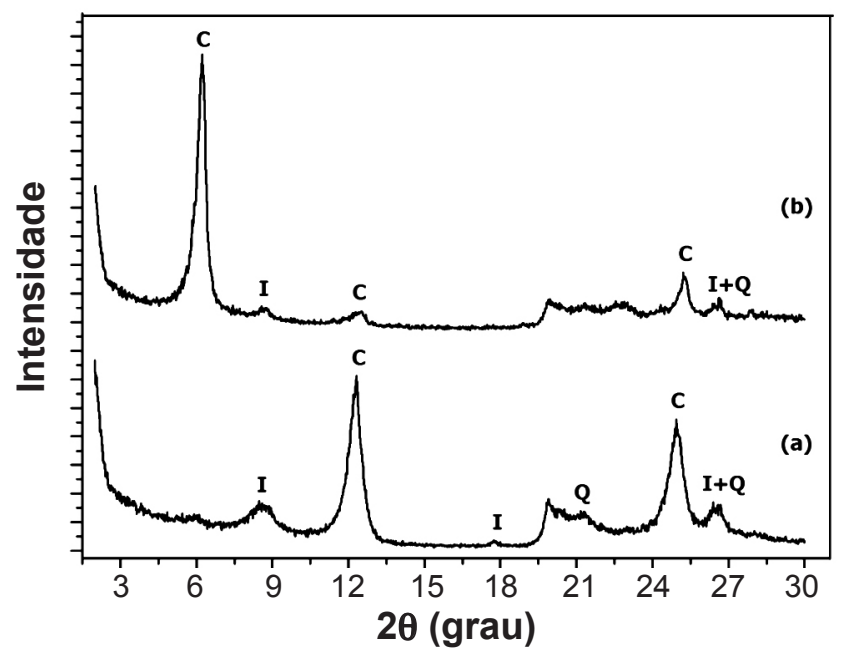

Figura 1: Difratogramas de raios $\mathrm{X}$ da amostra natural (a) e intercalada (b): caulinita (C), ilita (I) e quartzo (Q).

[Figure 1: X-ray diffraction patterns of natural sample (a) and intercalated (b): kaolinite $(C)$, illite $(I)$ and quartz $(Q)$. foi deslocado em direção à ângulos mais baixos, após a intercalação, como pode ser visto na Fig. 1b. Com o desvio do ângulo de reflexão, observou-se o deslocamento do pico $\mathrm{d}_{001}$ de $12,33^{\circ}$ para $6,33^{\circ}$, esta mudança corresponde a uma expansão da rede cristalográfica. Através da equação de Bragg $(\mathrm{n} \lambda=2 \mathrm{~d} \operatorname{sen} \theta)$ foi possível calcular o espaçamento basal da amostra natural e intercalada, que apresentaram os valores de $(7,16 \AA)$ e $(13,93 \AA)$, respectivamente, o que demonstra um aumento do espaçamento basal na ordem de 6,77 ̊̊. Isto aconteceu devido ao alojamento das moléculas do acetato de potássio no espaço interlamelar da caulinita. O processo da "moagem" provocou uma desestruturação do material, acarretando uma diminuição da intensidade dos picos na região de 20 a $27^{\circ}$. A água adicionada durante a moagem promove uma lavagem parcial do acetato intercalado e uma delaminação ou esfoliação da matriz, o que contribui com a desordem do material [7].

Observou-se ainda no difratograma da amostra intercalada (Fig. 1b) a presença do pico no ângulo $12,33^{\circ}$

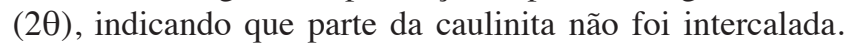
$\mathrm{O}$ grau de intercalação pode ser calculado por meio das intensidades dos picos relacionados ao plano basal 001 das amostras de acordo com a equação B $[6,16,17]$.

$\%$ intercalação: $\left\{\left[\mathrm{I}(001)_{\text {complexo }}\right] /\left[\mathrm{I}(001)_{\text {complexo }}+\mathrm{I}(001)_{\text {caulinita }}\right]\right\}$

Onde: $\mathrm{I}(001)_{\text {complexo }}$ e $\mathrm{I}(001)_{\text {caulinita }}$ são as intensidades dos picos relacionados ao plano basal 001 da caulinita intercalada e não intercalada, respectivamente. Portanto, o grau de intercalação calculado foi de $61,28 \%$ da caulinita intercalada. A intercalação do acetato de potássio na argila ocorre através da ponte de hidrogênio do íon acetato $\mathrm{CH}_{3} \mathrm{COO}^{-}$com os grupos $\mathrm{OH}$ da superfície interna da folha octaédrica $\mathrm{Al}-\mathrm{OH}$ e, também, com os átomos de $\mathrm{O}$ da folha tetraédrica O-Si-O [18].

Os resultados da análise química das amostras encontramse expostos na Tabela I, de modo que para a amostra natural a soma de sílica e alumínio na forma de óxidos corresponde a mais de $75 \%$ em peso. Sendo que sua composição química é característica de argilas cauliníticas contendo como minerais acessórios quartzo, mica e minerais ferrosos.

Os resultados da análise química da amostra intercalada possibilitaram analisar a quantidade de potássio introduzido na estrutura da mesma. Observa-se que o valor de $\mathrm{K}_{2} \mathrm{O}$ e perda ao fogo aumentaram com a introdução da molécula do acetato de potássio na estrutura da amostra, enquanto os conteúdos dos demais elementos decaíram, levando à conclusão de que ocorreu a intercalação do acetato de potássio.

Tabela I - Análise química por fluorescência de raios X das amostras natural e intercalada.

[Table I - Chemical analysis by X-ray fuorescence of natural and intercalated samples.]

\begin{tabular}{lccccccc}
\hline Constituintes \% & $\mathbf{S i O}_{2}$ & $\mathbf{A l}_{2} \mathbf{O}_{3}$ & $\mathbf{K}_{2} \mathbf{O}$ & $\mathbf{F e}_{2} \mathbf{O}_{3}$ & $\mathbf{M g O}$ & Outros & L.O.I \\
\hline Amostra natural & 41,97 & 33,76 & 1,38 & 6,56 & 2,17 & 1,47 & 12,69 \\
Amostra intercalada & 28,67 & 24,17 & 14,12 & 4,60 & 1,13 & 1,02 & 26,29 \\
\hline
\end{tabular}


A Fig. 2a mostra a micrografia da amostra natural, onde se verifica a presença de cristais com formato lamelar, e no formato do tipo "booklets", características do empilhamento dos cristais de caulinita [19]. A Fig. 2b mostra a micrografia da amostra intercalada com acetato de potássio. A imagem revela claramente que os cristalitos apresentam cantos e bordas um pouco menos definidos, mais arredondados e aglomerados, ligados uns aos outros formando uma espécie de matriz compacta, consequência do procedimento de moagem da reação de intercalação.

Nos espectros de infravermelho da amostra natural (Fig. 3a) são notados bandas características do argilomineral caulinita em 472, 541 e $1011 \mathrm{~cm}^{-1}$ [20]. As bandas em 910, $1026,1114 \mathrm{~cm}^{-1}$ são atribuídas ao estiramento vibracional do Si-O, enquanto as bandas em 668, 750 e $787 \mathrm{~cm}^{-1}$ estão relacionadas ao estiramento vibracional do grupo O-Al$\mathrm{OH}$. Na região entre 3600 e $3700 \mathrm{~cm}^{-1}$ é observado duas bandas bem definidas, sendo que a banda em $3688 \mathrm{~cm}^{-1}$ é característica do estiramento do grupo $\mathrm{OH}$. E a banda em $3619 \mathrm{~cm}^{-1}$ está relacionada à hidroxila interna orientada quase paralelamente à direção da camada (001), apontada na
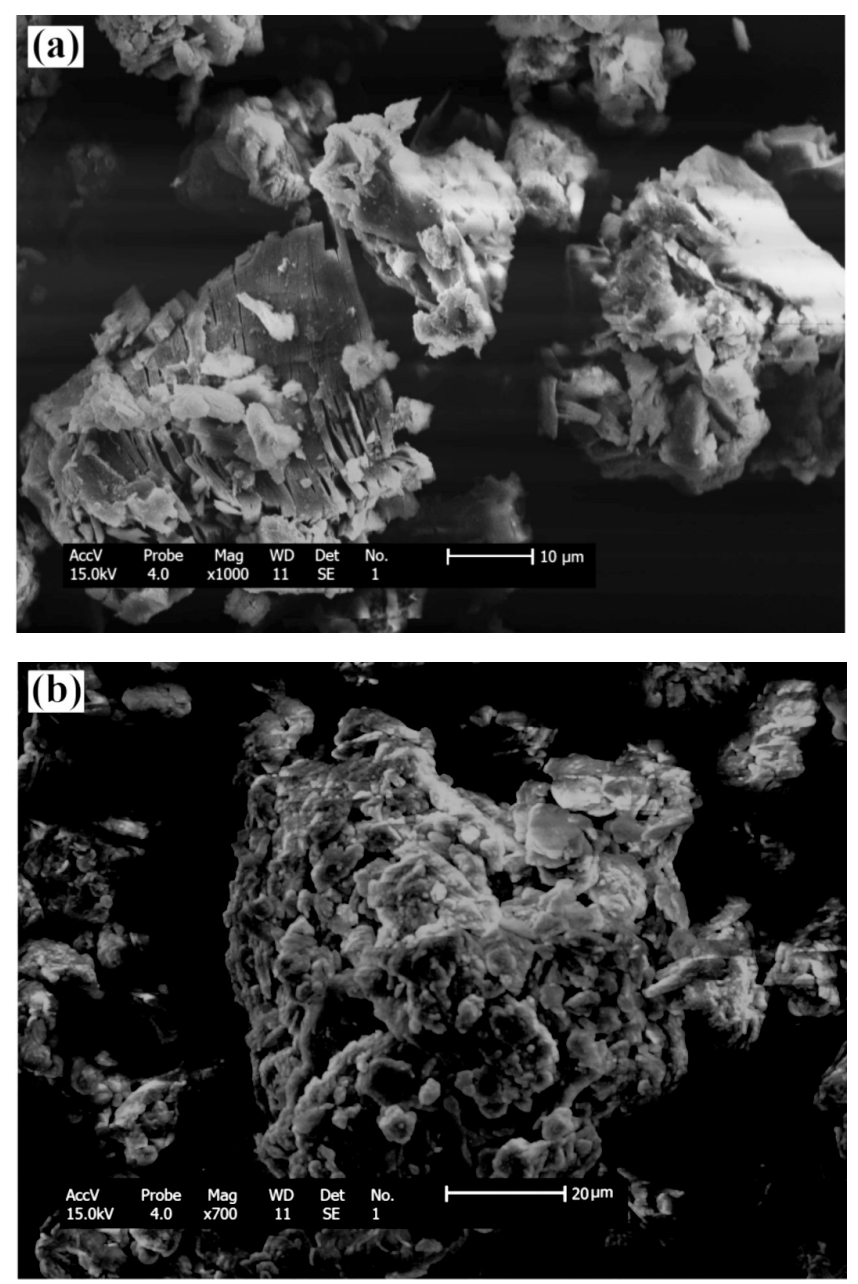

Figura 2: Imagens de micrografia eletrônica de varredura da amostra natural (a) e intercalada (b).

[Figure 2: Images of scanning electron microscopy of the natural sample (a) and intercalated sample (b).]

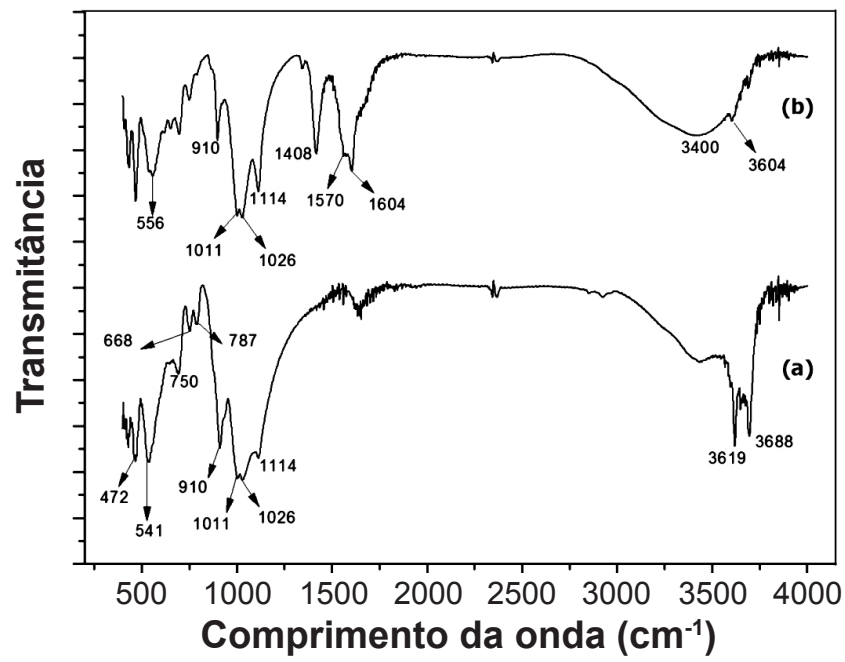

Figura 3: Espectros de infravermelho da amostra natural (a) e intercalada (b).

[Figure 3: Infrared spectra of natural sample (a) and intercalated (b).]

direção da vacância do sítio octaédrico e, geralmente, não muito influenciada pelas reações de modificação interlamelar [21]. Os espectros de infravermelho da amostra intercalada (Fig. 3b) mostraram bandas adicionais na região de $1408 \mathrm{e}$ $1604 \mathrm{~cm}^{-1}$ atribuídas as vibrações de estiramento simétricas e vibrações anti-simétricas do $\mathrm{CH}_{3} \mathrm{COO}^{-}$. Uma ampla banda com transmitância mínima em 3400 e duas bandas em 1570 e $3604 \mathrm{~cm}^{-1}$ são observadas no espectro da amostra intercalada e podem ser atribuídas ao estiramento do grupo $\mathrm{OH}$ da água absorvida pelo sal do acetato [22]. Outras bandas em 1408, $1114 \mathrm{e} 556 \mathrm{~cm}^{-1}$, presentes neste espectro foram (atribuidas à formação de fortes ligações de hidrogênio entre a matriz da caulinita e os íons acetato [23].

\section{Estudo da remoção do corante azul de metileno}

Um dos fatores mais importantes em estudos de adsorção é o efeito do $\mathrm{pH}$ no meio. As quantidades de MB adsorvido pelas amostras natural e intercalada foram monitoradas numa faixa de $\mathrm{pH}$ de 2 a 10 e temperatura de $25 \pm 1^{\circ} \mathrm{C}$. Para caulinita natural $(\mathrm{CN})$ a adsorção máxima ocorreu em pH 9,0. E para caulinita intercalada $(\mathrm{CM})$ o pH favorável para adsorção do corante MB foi 7,0. Assim, nos estudos posteriores foram fixados o $\mathrm{pH}$ de 9,0 para caulinita natural e pH 7,0 para caulinita intercalada.

Os parâmetros da equação dos modelos de equilíbrio empregados muitas vezes fornecem informações sobre o mecanismo de adsorção, as propriedades da superfície e afinidade do adsorvente. As isotermas de adsorção do corante sobre a caulinita natural, Fig. 4a, e caulinita intercalada, Fig. 4b, foram plotadas, utilizando os modelos de isotermas de Langmuir, Freundlich e Sips. Os parâmetros foram estimados por regressão não-linear, e os coeficientes de determinação encontrados para cada modelo apresentaram valores maiores que 0,95 . Como pode ser visto na Tabela II, para ambas as amostras, o modelo que melhor descreveu 

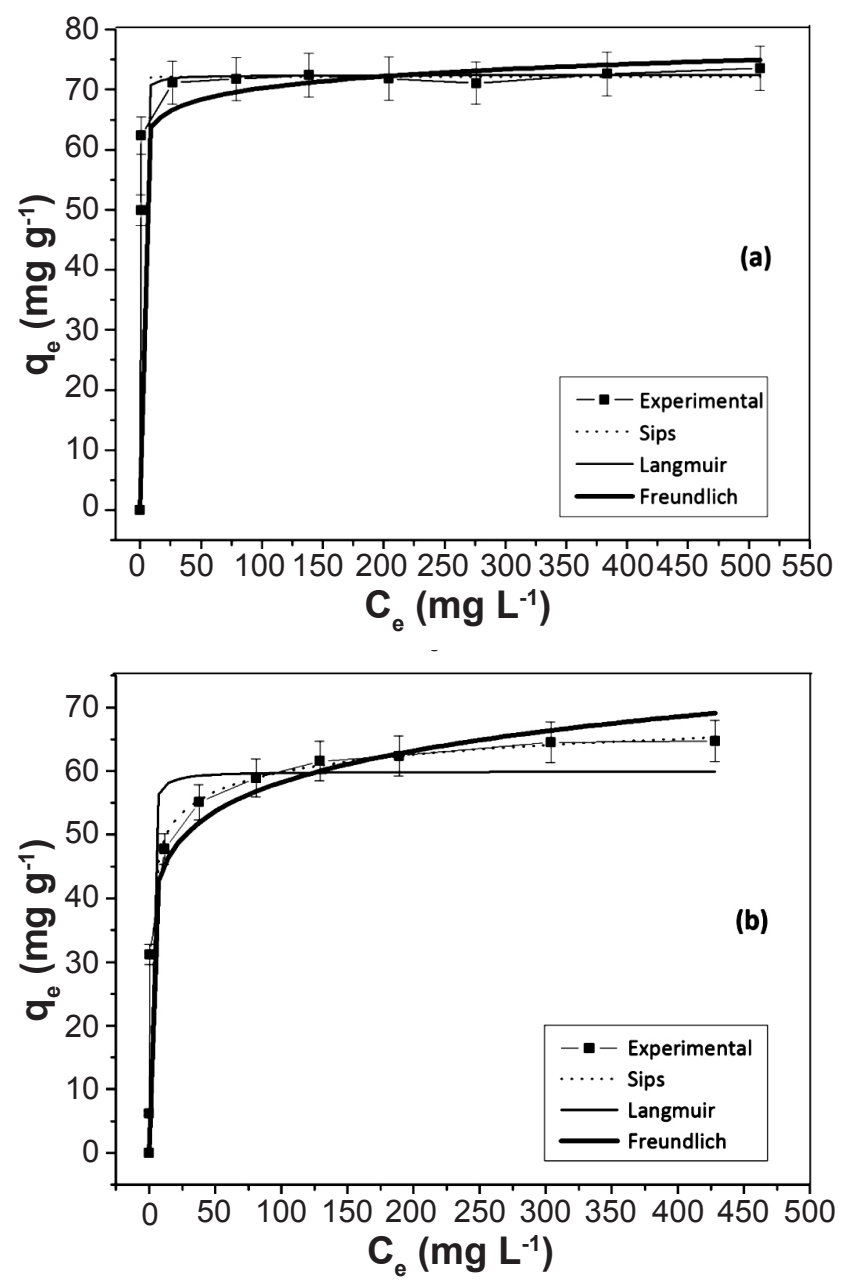

Figura 4: Modelos de isotermas de adsorção do corante MB em solução aquosa, caulinita natural (a) e caulinita intercalada (b). Condições de análises: Temperatura $25 \pm 1{ }^{\circ} \mathrm{C}, \mathrm{pH}=9,0$ para caulinita natural e $\mathrm{pH}=7,0$ para caulinita intercalada, tempo de contato $4 \mathrm{~h}$.

[Figure 4: Models of adsorption isotherms of $M B$ dye in aqueous solution, natural kaolinite (a) and intercalated kaolinite (b). Conditions of analyzes: Temperature $25 \pm 1^{\circ} \mathrm{C}, \mathrm{pH}=9.0$ for natural kaolinite and $\mathrm{pH}=7.0$ for kaolinite intercalated, contact time 4 h.]

o processo de adsorção foi o de Sips, pois apresentou um menor valor de $\chi^{2}$ e um valor de $\mathrm{R}^{2}$ mais próximo de 1 .

As diferenças dos valores entre os $\chi^{2}$ de Sips em relação aos outros modelos de isoterma são bastante expressivos, indicando que os modelos de Freundlich e Langmuir, não se adequaram devidamente a isoterma experimental (Tabela II). O modelo de Sips é uma combinação dos modelos de Langmuir e Freundlich, ou seja, em baixas concentrações do adsorbato é efetivamente reduzida para isoterma de Freundlich, não obedecendo à lei de Henry. Em altas concentrações do adsorbato, o modelo prevê uma capacidade de adsorção em monocamada característica da isoterma de Langmuir [24]. Considerando que os dados de equilíbrio foram melhores ajustados ao modelo de isoterma de Sips, as capacidades máximas de adsorção foram de 72,14 e 79,34 mg $\mathrm{g}^{-1}$ para a caulinita natural e intercalada, respectivamente. Os dados experimentais da caulinita intercalada, ainda que não seja visível no gráfico (Fig. 4b), não atingiram o equilíbrio. O modelo de Sips prevê uma adsorção além dos dados experimentais quando os valores de $\mathrm{q}_{\mathrm{e}}$ ainda não atingiram um "plateau" constante, por isso, os valores de $q_{m}$ de Sips citados são superiores aos dados de $\mathrm{q}_{\mathrm{e}}$ obtidos. Quando se compara a capacidade máxima de adsorção das amostras de caulinita deste trabalho com outras da literatura [25], nota-se que as estudadas apresentam boa capacidade de adsorção do corante azul de metileno. Esse fato pode estar relacionado à presença do argilomineral ilita na composição da caulinita estudada, que é um filossilicato do tipo 2:1, cuja propriedade pode auxiliar no processo de adsorção.

Com a finalidade de conhecer as características da cinética de adsorção da interação do corante MB com as amostras, foram aplicados três modelos cinéticos: pseudo-primeira ordem, pseudo-segunda ordem e Avrami. Considerando o mecanismo de adsorção, os modelos cinéticos foram aplicados aos dados experimentais para os sistemas MB/ caulinita natural e $\mathrm{MB} /$ caulinita intercalada. Foram construídos gráficos de $\mathrm{q}_{\mathrm{e}}\left(\mathrm{mg} \mathrm{g}^{-1}\right)$ vs $\mathrm{t}$ (min) e plotados os modelos nos dados experimentais das amostras. De acordo com os gráficos obtidos, o tempo mínimo necessário para o sistema atingir o equilíbrio é em torno de $90 \mathrm{~min}$ para a caulinita natural e $120 \mathrm{~min}$ para caulinita intercalada, conforme mostrada nas Figs. 5a e 5b.

Como pode ser visto o modelo de Avrami foi o qual os dados da caulinita natural e da caulinita intercalada melhor se ajustaram. Essa avaliação foi feita por meio da comparação entre os coeficientes de determinação $\left(\mathrm{R}^{2}\right)$ e pelo menor valor do chi-quadrado $\left(\chi^{2}\right)$ para ambas as amostras, conforme apresentada na Tabela III. Além disso, verificou-se que os valores encontrados de $\mathrm{q}_{\mathrm{e}}$ do modelo de Avrami estavam mais próximos dos valores de $\mathrm{q}_{\mathrm{e}}$ experimentais, principalmente da caulinita intercalada, quando comparado com os outros

Tabela II - Parâmetros das isotermas de adsorção do corante $\mathrm{MB}$ das amostras. Condições: Temperatura $25 \pm 1^{\circ} \mathrm{C}, \mathrm{pH}=9,0$ para caulinita natural $(\mathrm{CN})$ e $\mathrm{pH}=7,0$ para caulinita intercalada $(\mathrm{CM})$, tempo de contato $4 \mathrm{~h}$.

[Table II - Parameters of adsorption isotherms of the dye MB of samples. Conditions: temperature $25 \pm 1{ }^{\circ} \mathrm{C}, \mathrm{pH}=9.0$ for natural kaolinite (CN) and pH 7.0 for kaolinite intercalated (CM), time contact $4 \mathrm{~h}$.

\begin{tabular}{cccccccccccccccc}
\hline \multicolumn{1}{c}{ Amostras } & \multicolumn{3}{c}{ Langmuir } & \multicolumn{4}{c}{ Freundlich } & \multicolumn{4}{c}{ Sips } \\
\hline & $\mathrm{q}_{\mathrm{m}}$ & $\mathrm{k}_{\mathrm{L}}$ & $\mathrm{R}^{2}$ & $\chi^{2}$ & $\mathrm{k}_{\mathrm{F}}$ & $\mathrm{n}_{\mathrm{F}}$ & $\mathrm{R}^{2}$ & $\chi^{2}$ & $\mathrm{q}_{\mathrm{m}}$ & $\mathrm{k}_{\mathrm{s}}$ & $\mathrm{n}_{\mathrm{s}}$ & $\mathrm{R}^{2}$ & $\chi^{2}$ \\
$\mathrm{CN}$ & 72,45 & 4,71 & 0,994 & 3,225 & 58,40 & 24,96 & 0,977 & 13,042 & 72,14 & 8,32 & 0,43 & 0,998 & 0,679 \\
$\mathrm{CM}$ & 59,97 & 2,13 & 0,960 & 26,421 & 33,85 & 8,49 & 0,982 & 11,454 & 79,34 & 0,82 & 3,50 & 0,999 & 0,592 \\
\hline
\end{tabular}



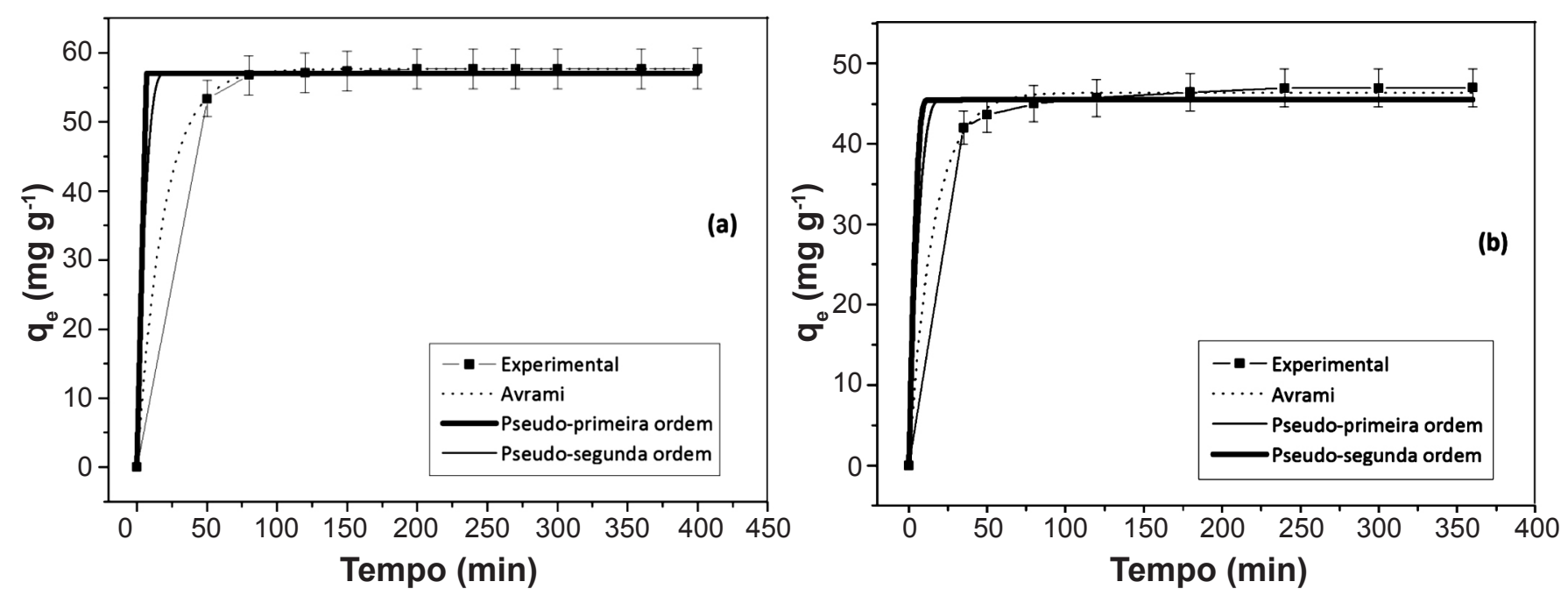

Figura 5: Modelos cinéticos de adsorção do corante MB em solução aquosa, caulinita natural (a) e caulinita intercalada (b). Condições: Temperatura $25 \pm 1^{\circ} \mathrm{C}, \mathrm{pH}=9,0$ para caulinita natural e $\mathrm{pH}=7,0$ para caulinita intercalada, concentração do corante $313,75 \mathrm{mg} \mathrm{L}{ }^{-1}$. [Figure 5: Kinetic models adsorption of the dye MB in aqueous solution, natural kaolinite (a) and intercalated kaolinite (b). Conditions: Temperature $25 \pm 1^{\circ} \mathrm{C}, \mathrm{pH}=9.0$ for natural kaolinite and $\mathrm{pH}=7.0$ for kaolinite intercalated, concentration of dye $\left.313.75 \mathrm{mg} \mathrm{L}^{-1}.\right]$

Tabela III - Parâmetros cinéticos de adsorção do corante MB das amostras. Condições: Temperatura $25 \pm 1^{\circ} \mathrm{C}, \mathrm{pH}=$ 9,0 para caulinita natural $(\mathrm{CN})$ e $\mathrm{pH}=7,0$ para caulinita intercalada $(\mathrm{CM})$, concentração do corante $313,75 \mathrm{mg} \mathrm{L}^{-1}$.

[Table III - Kinetic parameters of the dye adsorption MB of samples. Conditions: temperature $25 \pm 1{ }^{\circ} \mathrm{C}, \mathrm{pH}=9.0$ for natural kaolinite $(C N)$ and $\mathrm{pH} 7.0$ for kaolinite intercalated $(\mathrm{CM})$, concentration of dye $313.75 \mathrm{mg} \mathrm{L^{-1 }}$.]

\begin{tabular}{ccccccccccccccc}
\hline Amostras & \multicolumn{1}{c}{ Pseudo-primeira ordem } & \multicolumn{4}{c}{ Pseudo-segunda ordem } & \multicolumn{4}{c}{ Avrami } \\
\hline & $\mathrm{q}_{\mathrm{e}}$ & $\mathrm{K}_{\mathrm{f}}$ & $\mathrm{R}^{2}$ & $\chi^{2}$ & $\mathrm{q}_{\mathrm{e}}$ & $\mathrm{K}_{\mathrm{s}}$ & $\mathrm{R}^{2}$ & $\chi^{2}$ & $\mathrm{q}_{\mathrm{e}}$ & $\mathrm{K}_{\mathrm{AV}}$ & $\mathrm{n}_{\mathrm{AV}}$ & $\mathrm{R}^{2}$ & $\chi^{2}$ \\
$\mathrm{CN}$ & 57,04 & 2,06 & 0,994 & 1,812 & 57,05 & 4,30 & 0,994 & 1,809 & 57,70 & 0,04 & 1,28 & 0,999 & 0,050 \\
$\mathrm{CM}$ & 45,46 & 0,33 & 0,987 & 3,312 & 45,48 & 3,75 & 0,987 & 3,304 & 46,36 & 0,04 & 1,32 & 0,997 & 0,632 \\
\hline
\end{tabular}

modelos cinéticos. O modelo cinético de Avrami descreve reações de ordens fracionárias, sugerindo que os processos de interação sofrem mudanças no mecanismo e na velocidade de reação durante o tempo analisado $[26,27]$.

\section{CONCLUSÃO}

A união dos dados da caracterização juntamente com os dados de literatura, revelou que a amostra de Bom Jardim de Goiás, GO é composta essencialmente pelo argilomineral caulinita. A intercalação do acetato de potássio foi satisfatória, sendo evidenciado pelas caracterizações, principalmente pelas análises de difração de raios $\mathrm{X}$ e infravermelho. $\mathrm{O}$ corante interagiu com ambas as amostras (natural e intercalada) na interface sólido/líquido, quando suspenso em solução aquosa. As isotermas de equilíbrio do MB foram obtidas e melhor se ajustaram ao modelo de Sips, uma vez que os modelos de Langmuir e Freundlich não se adequaram aos dados experimentais, devido os elevados valores do chi-quadrado. Com base nos resultados obtidos, pode-se inferir que as amostras apresentaram eficiência na adsorção do corante azul de metileno, quando comparado aos resultados obtidos em outros trabalhos, sendo que as capacidades máximas de adsorção da caulinita natural e caulinita intercalada foram de 72,14 e 79,34 mg.g ${ }^{-1}$, respectivamente. Três modelos cinéticos foram aplicados para ajustar dados experimentais, sendo que o modelo de Avrami apresentou um maior valor do coeficiente de determinação e, deste modo, foi o que melhor se ajustou para ambas as amostras. Dessa forma, o tempo mínimo necessário para o sistema atingir o equilíbrio foi de $90 \mathrm{~min}$ para a caulinita natural e 120 min para caulinita intercalada. Portanto, as amostras de caulinita natural e intercalada com acetato de potássio, oriundas da região de Bom Jardim, GO, possuem uma boa capacidade de adsorção e podem ser eficazes na remoção do corante azul de metileno em meios aquosos.

\section{AGRADECIMENTOS}

À CAPES e ao CNPq pelo suporte financeiro.

\section{REFERÊNCIAS}

[1] K. M. Parida, S. Sahu, K. H. Reddy, P. C. Sahoo, Ind \& Eng. Chem. Res. 50 (2010) 843.

[2] T. Liu, Y. Li, Q. Du, J. Sun, Y. Jiao, G. Yang, Z. Wang, Y. Xia, W. Zhang, K. Wang, H. Zhu, D. Wu, Colloids and 
Surfaces B: Biointerfaces. 90 (2012) 197.

[3] K. G. Bhattacharyya, S. S. Gupta, Colloids and Surfaces A: Physicochem. Eng. Aspects 277 (2006) 191.

[4] G. Annadurai, R. S. Juang, D. J. Lee, J. Hazardous Mater. 92 (2002) 263.

[5] J. E. Gardolinski, P. P. Zamora, F. Wypych, J. Colloid Interface Sci. 211 (1999) 137.

[6] H. Cheng, Q. Liu, J. Yang, X. Du, R. L. Frost, Appl. Clay Sci. 50 (2010) 476.

[7] J. E. Gardolinski, Compostos de intercalação derivados da caulinita, Diss. Mestrado, Programa de Pós-Graduação em Engenharia, UFPR, Curitiba, PR (2001).

[8] M. A. Martín-Lara, I. L. R. Rico, I. C. A. Vicente, G. B. García, M. C. Hoces, Desalination 256 (2010) 58.

[9] S. J. Allen, Q. Gan, R. Matthews, P. A. Johnson, Bioresource Technology 88 (2003) 143.

[10] A. Dąbrowski, Adv. Colloid Interface Sci. 93 (2001) 135.

[11] B. Royer, N. F. Cardoso, E. C. Lima, J. C. P. Vaghetti, N. M. Simon, T. Calvete, R. C. Veses, J. Hazardous Mater. 164 (2009) 1213.

[12] Y. S. Ho, G. McKay, Chem. Eng. J. 70 (1998) 115.

[13] Y. S. Ho, G. McKay, Process Biochem. 34 (1999) 451.

[14] E. C. N. Lopes, F. S. C. Anjos, E. F. S. Vieira, A. R. Cestari, J. Colloid Interface Sci. 263 (2003) 542.

[15] D. M. Moore, R. C. Reynolds, X-ray diffraction and the identification and analysis of clay minerals, $2^{\text {nd }}$ Ed. Oxford
Univ. Press, New York, EUA (1997) 378.

[16] M. D. R. Cruz , F. I. F. Duro, Clay Minerals 34 (1999) 565.

[17] R. L. Frost, J. Kristof, E. Horvath, J. T. Kloprogge, J. Colloid Interface Sci. 214 (1999) 109.

[18] R. L. Frost, J. Kristof, G. N. Paroz, T. H. Tran, J. T. Kloprogge, J. Colloid Interface Sci. 204 (1998) 227.

[19] A. A. B. Maia, E. Saldanha, R. S. Angélica, C. A. G. Souza, R. F. Neves, Cerâmica 53 (2007) 319.

[20] H. Xu, M. Wang, Q. Liu, D. Chen, H. Wang, K. Yang, H. Lu, R. Zhang, S. Guan, J. Phys. Chem. Solids. 72 (2011) 24.

[21] Y. Turhan, M. Dogan, M. Alkan, Ind. \& Eng. Chem. Res. 49 (2010) 1503.

[22] R. L. Ledoux, J. L. White, J. Colloid Interface Sci. 21 (1966) 127.

[23] R. L. Frost, T. H. Tran, Kristof, Vibrational Spectroscopy 13 (1997) 175.

[24] Y. S. Ho, J. F. Porter, G. McKay, Water Air and Soil Pollution 141 (2002) 1.

[25] D. Ghosh, K. G. Bhattacharyya, Appl. Clay Sci. 20 (2002) 295.

[26] A. R. Cestari, E. F. S. Vieira, A. G. P. Santos, J. A. Mota, V. P. Almeida, J. Colloid Interface Sci. 280 (2004) 380.

[27] J. Wang, H. C. Kou, X. F. Gu, J. S. Li, L. Q. Xing, R. $\mathrm{Hu}, \mathrm{L}$. Zhou, Mater. Lett. 63 (2009) 1153.

(Rec. 20/02/2012, Rev. 07/08/2012, Ac. 04/10/2012) 